\title{
Spherically symmetric solutions in higher-derivative theories of gravity
}

\author{
G. Rodrigues-da-Silva* \\ Departamento de Física, Universidade Federal do Rio Grande do Norte, \\ Campus Universitário, s/n - Lagoa Nova, CEP 59072-970, Natal, Rio Grande do Norte, Brazil \\ L. G. Medeiros ${ }^{\dagger}$ \\ Escola de Ciências e Tecnologia, Universidade Federal do Rio Grande do Norte, \\ Campus Universitário, s/n - Lagoa Nova, CEP 59072-970, Natal, Rio Grande do Norte, Brazil
}

(Dated: August 4, 2020)

\begin{abstract}
Higher-order theories of gravity have received much attention from several areas including quantum gravity, string theory and cosmology. This paper proposes a higher-order gravity whose action includes all curvature scalar terms up to the second-order corrections of general relativity, namely, $R^{2}, R^{3}$ and $R \square R$. Then, we explore spherically symmetric and static solutions in the weak-field regime and black holes context. All solutions in the weak-field regime due to a point mass are deduced, and by making a stability analysis of these solutions, we restrict them to Yukawa-type solutions. In regard to black hole solutions, we use the Lichnerovicz method to investigate the possibility of existence of non-Schwarzschild black holes. The results obtained show that non-Schwarzschild solutions might exist. However, for reasonable values of the parameters of the theory, its horizon radii are extremely small making macroscopic black holes different from Schwarzschild unfeasible.
\end{abstract}

\section{INTRODUCTION}

General relativity (GR) has still been our standard theory for describing gravity. This is due to its immense predictive power, which even after a century it continues to be corroborated by observations, namely, direct detections of gravitational waves from the binary black hole and neutron star mergers [1-3].

Despite the great success of GR, extensions to it have received much attention from several areas including highenergy physics, e.g. string theory, the cosmology of the early and late universe, and astrophysics.

From a theoretical point of view, one of the limitations of GR is the fact that it is non-renormalizable, making its quantization problematic. In this sense, a very relevant kind of extended theory of gravity is the higher-order one, in which the Einstein-Hilbert action is supplemented by higher-order curvature terms. From this perspective, GR is viewed as an effective low-energy theory that requires higher-order corrections as we increase the energy scale [4]. Its relevance is justified since in order to construct a quantum theory of gravity it was found that higher-order terms contribute to the renormalizability of the theory [5]. In fact, K. S. Stelle showed that by adding to Einstein-Hilbert action all relevant curvature terms up to the second-order, one obtains a perturbatively renormalizable system, however, at the price of introducing ghost-type instabilities, which present themselves as states with negative energy or negative norm [6]. Classically, as presented by Ostrogradski, due to the presence of higher-order derivatives, in general, the Hamiltonian of the system becomes not bounded from below [7]. This is the known tension between renormalizability and unitarity [8-10]. Allying one to another represents one of the biggest problems in quantum gravity, and

\footnotetext{
*gesiel.fisica@gmail.com

† leogmedeiros@ect.ufrn.br
}

that's one of the reasons why we still have no fully consistent model capable of describing gravity in the ultraviolet (UV) regime.

In order to construct a quantum theory of gravity, addressing the renormalizability problem, we highlight two interesting approaches to extensions of GR. One of those is based on gravities constructed by the Einstein-Hilbert action supplemented by terms involving a polynomial function of the covariant d'Alembertian operator, such as $R F_{1}(\square) R$ and $R_{\mu \nu} F_{2}(\square) R^{\mu \nu}[8,11]$. A theory in which corrections of this type are included has a finite number of curvature derivatives and is (super-)renormalizable and local. It is also worth mentioning that in this approach the ghosts problem can be addressed through the quantization process $a$ la Lee-Wick $[12,13]$. The other approach involves replacing polynomial with non-polynomial operator functions of the covariant d'Alembertian, such as $R \exp (\square) R$ and $R_{\mu \nu} \exp (\square) R^{\mu \nu}$, that makes a theory with infinite curvature derivatives, (super)renormalizable, non-local and ghost-free at the tree-level [14-17].

Although we cite renormalizability and unitarity as strong theoretical motivations driving the search for higher-order theories of gravity, we are not interested in proposing a model in the quantum gravity scenario. Instead, our interest lies in classical models of gravity motivated by the context of effective theory, in which the included higher-order curvature terms become more relevant in a given energy scale. Some examples of works that consider higher-order gravities including curvature derivatives terms are: Refs. [18-20], in which the authors analyze low-energy effects; Refs. [21, 22], in which they develope the gauge formulation and a study of equivalence between theories, respectively; and Refs. [23-25], in which the authors propose an extension to the Starobinsky's inflationary model.

For the sake of simplicity, the gravity treated here is built only with the curvature scalar $R$ and its derivatives we will not include, for example, terms like $R_{\mu \nu} R^{\mu \nu}$ or $R_{\mu \nu} F_{2}(\square) R^{\mu \nu}$. It is characterized by including all correc- 
tion terms up to the second-order, that is, quadratic and cubic curvature correction terms involving the curvature scalar, i.e. $R^{2}, R^{3}$ and $R \square R$. This implies sixth-order field equations for the metric. Thus, the most general gravitational action up to the second-order and containing only the curvature scalar is given by

$$
\begin{aligned}
S=\frac{M_{P}^{2}}{2} \int d^{4} x \sqrt{-g} & \left(R+\frac{1}{2 \kappa_{0}} R^{2}+\frac{\alpha_{0}}{3 \kappa_{0}^{2}} R^{3}-\frac{\beta_{0}}{2 \kappa_{0}^{2}} R \square R\right)+ \\
& +\int d^{4} x \sqrt{-g} \mathcal{L}_{m}
\end{aligned}
$$

in which $M_{P}$ is the reduced Planck mass, so that $M_{P}^{2} \equiv$ $(8 \pi G)^{-1}$, and $\kappa_{0}, \alpha_{0}$ and $\beta_{0}$ are parameters of the theory, $\kappa_{0}$ being the only dimensional one (squared mass). The quantity $\mathcal{L}_{m}=\mathcal{L}_{m}\left(\psi, g_{\mu \nu}\right)$ represents the matter Lagrangian, which encapsulates the coupling of gravitation with the matter fields $\psi$.

One of the fundamental aspects in which the model defined by Eq. (1) can be addressed is in the study and analysis of its solutions. In the late 1970s, spherically symmetric solutions of the gravity with all quadratic curvature corrections were studied by Stelle [26]. In that paper, the weak-field regime of the theory became well established: each of the massive modes contributes to a decreasing Yukawa-type term, while the usual non-massive spin-2 mode contributes to the Newtonian potential. In addition, a full analysis was also performed by assuming a spherically symmetric and static metric in Schwarzschild coordinates, and by taking an expansion in Frobenius series around the origin of the two radial functions of the metric. However, as justified in Ref. [27], at that pre computational algebra time, it was not possible to have a complete view of the picture. This subject has recently been revived when, using Lichnerovicz and Israel type no-hair theorems, assuming the existence of a horizon $r_{h}$, along with asymptotic flatness at infinity, William Nelson has shown that not only the curvature scalar $R$ but also the Ricci tensor $R_{\mu \nu}$ would disappear outside the horizon [28]. This would mean that the Schwarzschild solution was the only spherically symmetric static solution of the analyzed gravity. On the other hand, it was shown in Ref. [29] that signal errors were made in the analysis of the traceless part, so that the Ricci tensor $R_{\mu \nu}$ would not disappear in the exterior region. This was an indication that non-Schwarzschild solutions might exist. In fact, families of non-Schwarzschild solutions were found numerically by Ref. [29], and then other papers confirmed these results using analytical [30, 31] and semi-analytical [32] approaches.

In this paper, we explore spherically symmetric and static solutions in the context of the weak-field regime and black hole solutions. Initially in Section II, we present the field equations of the gravity (1) and then we deduce all solutions in the weak-field regime due to a point mass. Similar to the analysis developed in Ref. [18], these solutions are obtained using an ansatz for the curvature scalar $R$ and for the gravitational potential $\phi$, and by establishing the boundary conditions, which are, a point mass at the origin and asymptotic flatness at the infinite. At first, among the solutions found are the decreasing Yukawa-type, oscillatory solutions and hybrid ones, involving Yukawa-type and oscillatory terms. However, in Subsection II A, a stability analysis of these solutions restricts the range of the theory parameters, discarding all solutions involving oscillatory terms. Regarding black hole solutions, in Section III, we use the Lichnerovicz method, similar to the found in Refs. [27, 28], to investigate the possibility of existence of exterior non-Schwarzschild solutions. At first, we use this method considering the full equations of the theory and, based on the Planck and inflationary energy scales, we estimate the order of magnitude of the horizon for the occurrence of such a non-Schwarzschild black hole. Then, in the Subsection III A, we explore the existence of deviations from the exterior Schwarzschild solution, and for that, we assume a continuous deformation of the curvature scalar. With this analysis we confirm in an alternative way the previous results of the Section III. Finally, our conclusions are presented in Section IV.

All over the work, the chosen metric signature is $(-,+,+,+)$, the Riemann tensor is written as $R_{\lambda \mu \nu}^{\kappa} \equiv$ $\partial_{\mu} \Gamma_{\lambda \nu}^{\kappa}-\partial_{\nu} \Gamma_{\lambda \mu}^{\kappa}+\Gamma_{\rho \mu}^{\kappa} \Gamma_{\lambda \nu}^{\rho}-\Gamma_{\rho \nu}^{\kappa} \Gamma_{\lambda \mu}^{\rho}$ and the Ricci tensor is defined as $R_{\lambda \nu} \equiv R_{\lambda \mu \nu}^{\mu}$.

\section{FIELD EQUATIONS AND WEAK-FIELD SOLUTIONS}

The field equations in the metric formalism associated with the action (1) can be written as

$$
\begin{aligned}
R_{\mu \nu}-\frac{1}{2} g_{\mu \nu} R+ & \frac{1}{\kappa_{0}}\left[R\left(R_{\mu \nu}-\frac{1}{4} g_{\mu \nu} R\right)-\nabla_{\mu} \nabla_{\nu} R+g_{\mu \nu} \square R\right]+\frac{\alpha_{0}}{\kappa_{0}^{2}}\left[R^{2}\left(R_{\mu \nu}-\frac{1}{6} g_{\mu \nu} R\right)-\nabla_{\mu} \nabla_{\nu} R^{2}+g_{\mu \nu} \square R^{2}\right]+ \\
& +\frac{\beta_{0}}{\kappa_{0}^{2}}\left[\nabla_{\mu} \nabla_{\nu} \square R+\frac{1}{2} \nabla_{\mu} R \nabla_{\nu} R-R_{\mu \nu} \square R-g_{\mu \nu}\left(\square^{2} R+\frac{1}{4} \nabla_{\alpha} R \nabla^{\alpha} R\right)\right]=8 \pi G T_{\mu \nu}, \quad(2)
\end{aligned}
$$

$$
\begin{aligned}
& \text { where } \square \equiv \nabla^{\mu} \nabla_{\mu} \text { and } \\
& \qquad T_{\mu \nu} \equiv \frac{2}{\sqrt{-g}} \frac{\delta\left(\sqrt{-g} \mathcal{L}_{m}\right)}{\delta g^{\mu \nu}} .
\end{aligned}
$$

These are sixth-order field equations for the metric. As already said, the terms beyond Einstein-Hilbert in the field equations (2) become increasingly relevant in the UV regime and they are regulated by powers of $\kappa_{0}$ in the denominator. 
From this point forward until the end of the section, we focus on the weak-field limit analysis of the gravity (1). By following the approach developed in Ref. [18], we analyze in detail all possible solutions in the weak-field regime due to the higher-order corrections.

In the weak-field approximation, in which $g_{\mu \nu}=\eta_{\mu \nu}+h_{\mu \nu}$, with $\left|h_{\mu \nu}\right| \ll 1$, the field equations can be expressed as

$$
\begin{gathered}
R_{\mu \nu}^{l i n}-\frac{1}{2} \eta_{\mu \nu} R^{l i n}+\frac{1}{\kappa_{0}}\left(\eta_{\mu \nu} \square R^{l i n}-\partial_{\mu} \partial_{\nu} R^{l i n}\right)+ \\
+\frac{\beta_{0}}{\kappa_{0}^{2}}\left(\partial_{\mu} \partial_{\nu} \square R^{l i n}-\eta_{\mu \nu}^{2} \square R^{l i n}\right)=8 \pi G T_{\mu \nu},
\end{gathered}
$$

since we ignore all nonlinear terms in $h_{\mu \nu}$. In Eq. (4), the superscript lin indicates that the quantities are linearized. Moreover, in this regime the box operator simplifies to $\square=\partial^{\mu} \partial_{\mu}$. By taking its trace and considering an energy-momentum tensor $T^{\mu \nu}$ of a dust-like fluid, namely, $T^{\mu \nu}=\rho u^{\mu} u^{\nu}$, where $u^{0} \gg u^{i}$, we get

$$
R^{l i n}-\frac{3}{\kappa_{0}}\left(1-\frac{\beta_{0}}{\kappa_{0}} \square\right) \square R^{l i n}=8 \pi G \rho,
$$

which in a static regime reduces to

$$
R^{l i n}-\frac{3}{\kappa_{0}}\left(1-\frac{\beta_{0}}{\kappa_{0}} \nabla^{2}\right) \nabla^{2} R^{l i n}=8 \pi G \rho .
$$

On the other hand, by relating Eq. (6) to the component $\mu=$ $\nu=0$ of Eq. (4), in which the geodesic equation gives us $h_{00}=-2 \phi$, with $\phi$ representing the classical gravitational potential, we obtain

$$
\nabla^{2} \phi+\frac{1}{6} R^{l i n}=\frac{16}{3} \pi G \rho .
$$

The expression (6) represents a fourth-order differential equation for the curvature scalar $R^{l i n}$. Thus, considering a spherically symmetric distribution, in which $\rho=M \delta(r)$, it has four linearly independent solutions. However, if we impose the condition of asymptotic flatness at infinity, in which $R \rightarrow 0$, we reduce the number of solutions for only two. Similarly, the general solution of Eq. (7) consists of six linearly independent solutions, and the physical imposition of asymptotic flatness at infinity discards three of them.

In order to obtain the solutions of Eq. (6), we propose the ansatz

$$
R^{l i n}=\frac{b_{+}}{r} e^{-\frac{r}{l_{+}}}+\frac{b_{-}}{r} e^{-\frac{r}{l_{-}}},
$$

where $b_{+}, b_{-}, l_{+}$and $l_{-}$are constants to be determined and $\operatorname{Re}\left(l_{ \pm}\right) \geq 0$. Moreover, by considering a point mass $M$ at the origin, so that $\rho=M \delta(r)$, and using

$$
\nabla^{2}\left(\frac{1}{r} e^{-\frac{r}{l}}\right)=\frac{1}{r l^{2}} e^{-\frac{r}{l}}-4 \pi \delta(r),
$$

with

$$
\nabla^{2}\left(\frac{1}{r}\right)=-4 \pi \delta(r)
$$

we obtain for Eq. (6) the expression

$$
\begin{gathered}
\frac{b_{+}}{r} e^{-\frac{r}{l_{+}}}\left(1-\frac{3}{\kappa_{0}} \frac{1}{l_{+}^{2}}+\frac{3 \beta_{0}}{\kappa_{0}^{2}} \frac{1}{l_{+}^{4}}\right)+ \\
+\frac{b_{-}}{r} e^{-\frac{r}{l_{-}}}\left(1-\frac{3}{\kappa_{0}} \frac{1}{l_{-}^{2}}+\frac{3 \beta_{0}}{\kappa_{0}^{2}} \frac{1}{l_{-}^{4}}\right)+ \\
+\frac{12}{\kappa_{0}}\left[b_{+}+b_{-}-\frac{\beta_{0}}{\kappa_{0}}\left(\frac{b_{+}}{l_{+}^{2}}+\frac{b_{-}}{l_{-}^{2}}\right)\right] \pi \delta(r)+ \\
-\frac{12 \beta_{0}}{\kappa_{0}^{2}}\left(b_{+}+b_{-}\right) \pi \nabla^{2} \delta(r)=8 \pi G M \delta(r) .
\end{gathered}
$$

Due to the independence of the various terms in the previous expression, we then get the following relations:

$$
\begin{gathered}
b_{+}+b_{-}=0 \\
\frac{3}{\kappa_{0}}\left[b_{+}+b_{-}-\frac{\beta_{0}}{\kappa_{0}}\left(\frac{b_{+}}{l_{+}^{2}}+\frac{b_{-}}{l_{-}^{2}}\right)\right]=2 G M
\end{gathered}
$$

and

$$
l_{ \pm}^{4}-\frac{3}{\kappa_{0}} l_{ \pm}^{2}+\frac{3 \beta_{0}}{\kappa_{0}^{2}}=0 .
$$

The coefficientes $l_{ \pm}$can be obtained by solving the biquadratic equation (14). Thus

$$
l_{ \pm}=\sqrt{\frac{3}{2 \kappa_{0}}\left(1 \pm \sqrt{1-\frac{4 \beta_{0}}{3}}\right)}
$$

and since $b_{+}+b_{-}=0$, we can get from Eq. (13) that

$$
b_{+}=\frac{2 G M \kappa_{0}}{3} \frac{1}{\sqrt{1-\frac{4 \beta_{0}}{3}}}=-b_{-} .
$$

In the limit $\left|\beta_{0}\right| \rightarrow 0$, which represents the weak-field limit of $R^{2}$ gravity, we can write

$$
l_{+}=\sqrt{\frac{3}{\kappa_{0}}} \text { and } l_{-}=0 .
$$

Thus, for $\kappa_{0}>0$ the $l_{+}$is associated with Yukawa term due to $R^{2}$ correction and $l_{-}$makes the other exponential in the expression (8) negligible.

In order to obtain the solution of Eq. (7), we use again that $\rho=M \delta(r)$, the Eq. (8) for the curvature scalar $R^{l i n}$ and the ansatz

$$
\phi=-\frac{G M}{r}\left(1+a_{+} e^{-\frac{r}{l_{+}}}+a_{-} e^{-\frac{r}{l_{-}}}\right),
$$

in which $a_{+}$and $a_{-}$are constants to be determined.

By following the same procedure performed previously, we obtain

$a_{+}=\frac{1}{6}\left(\frac{1+\sqrt{1-\frac{4 \beta_{0}}{3}}}{\sqrt{1-\frac{4 \beta_{0}}{3}}}\right)$ and $a_{-}=-\frac{1}{6}\left(\frac{1-\sqrt{1-\frac{4 \beta_{0}}{3}}}{\sqrt{1-\frac{4 \beta_{0}}{3}}}\right)$. 
Thus, the generalization for the Newtonian potential is given by Eq. (18) with coefficients (15) and (19). It is interesting to evaluate the gravitational potential $\phi$ at the limit where $\beta_{0} \rightarrow$ 0 . In this case, we have

$$
a_{+}=\frac{1}{3}, \quad a_{-}=0 \text { and } l_{+}=\sqrt{\frac{3}{\kappa_{0}}}
$$

so that

$$
\phi=-\frac{G M}{r}\left(1+\frac{1}{3} e^{-\sqrt{\frac{\kappa_{0}}{3}} r}\right)
$$

In order to investigate the nature of the all possible solutions, we perform a case analysis of the relations (15). By defining the real positive quantities

$$
\begin{gathered}
A_{ \pm}=1 \pm \sqrt{1-\frac{4 \beta_{0}}{3}} \text { with } 0<\beta_{0}<3 / 4, \\
B_{ \pm}=\sqrt{1+\frac{4\left|\beta_{0}\right|}{3}} \pm 1 \text { with } \beta_{0}<0 \\
C_{ \pm}=\sqrt{\sqrt{\frac{4 \beta_{0}}{3}} \pm 1} \text { with } \beta_{0}>3 / 4
\end{gathered}
$$

all possible solutions can be summarized in Tables I and II:

Table I. This table shows the values obtained for $l_{ \pm}$in each of the intervals of $\beta_{0}$ for positive $\kappa_{0}$. Note that only the range $0<\beta_{0}<$ $3 / 4$ gives us real quantities for both $l_{ \pm}$.

\begin{tabular}{|c|c|c|}
\hline \multicolumn{3}{|c|}{$\kappa_{0}>0$} \\
\hline \hline$\beta_{0}$ & $l_{+}$ & $l_{-}$ \\
\hline $0<\beta_{0}<3 / 4$ & $\sqrt{\frac{3}{2 \kappa_{0}} A_{+}}$ & $\sqrt{\frac{3}{2 \kappa_{0}} A_{-}}$ \\
\hline$\beta_{0}<0$ & $\sqrt{\frac{3}{2 \kappa_{0}} B_{+}}$ & $i \sqrt{\frac{3}{2 \kappa_{0}} B_{-}}$ \\
\hline$\beta_{0}>3 / 4$ & $\sqrt{\frac{3}{4 \kappa_{0}}}\left(C_{+}+i C_{-}\right)$ & $\sqrt{\frac{3}{4 \kappa_{0}}}\left(C_{+}-i C_{-}\right)$ \\
\hline
\end{tabular}

Table II. This table shows the values obtained for $l_{ \pm}$in each of the intervals of $\beta_{0}$ for negative $\kappa_{0}$. None of them gives real values for both $l_{ \pm}$.

\begin{tabular}{|c|c|c|}
\hline \multicolumn{3}{|c|}{$\kappa_{0}<0$} \\
\hline \hline$\beta_{0}$ & $l_{+}$ & $l_{-}$ \\
\hline $0<\beta_{0}<3 / 4$ & $i \sqrt{\frac{3}{2\left|\kappa_{0}\right|} A_{+}}$ & $i \sqrt{\frac{3}{2\left|\kappa_{0}\right|} A_{-}}$ \\
\hline$\beta_{0}<0$ & $i \sqrt{\frac{3}{2\left|\kappa_{0}\right|} B_{+}}$ & $\sqrt{\frac{3}{2\left|\kappa_{0}\right|} B_{-}}$ \\
\hline$\beta_{0}>3 / 4$ & $\sqrt{\frac{3}{4 \mid \kappa_{0}}}\left(C_{-}-i C_{+}\right)$ & $\sqrt{\frac{3}{4\left|\kappa_{0}\right|}}\left(C_{-}+i C_{+}\right)$ \\
\hline
\end{tabular}

The $\kappa_{0}>0$ with $0<\beta_{0}<3 / 4$ is the only case where $l_{ \pm} \in \mathbb{R}$, implying Yukawa corrections $r^{-1} e^{-r / l_{ \pm}}$to the gravitational potential. On the other hand, $\kappa_{0}<0$ with $0<\beta_{0}<3 / 4$ is the only case where $l_{ \pm}$are pure imaginary, implying oscillatory corrections. In all other cases, $l_{ \pm} \in \mathbb{C}$, and the corrections contain Yukawa-type parts and oscillating ones.
It is interesting to note that the real parts $\operatorname{Re}\left(l_{ \pm}\right)$are always nonnegative. Thus, all the presented cases represent good solutions for $R$, since all of them are compatible with the established boundary conditions at the infinite. In some cases, as we can typically see when $\kappa_{0}>0$ and $\beta_{0}<0$, one solution has an oscillatory behavior, however, the factor $r$ in the denominator of (8) causes $R \rightarrow 0$ at infinity. Nevertheless, a relevant question is: are all the shown cases above physically feasible, or are there any intervals at which the parameters $\kappa_{0}$ and $\beta_{0}$ must be restricted? By investigating the stability of solutions to gravitational potential $\phi$, we will answer this question.

\section{A. Stability analysis}

In order to restrict the possible values that the parameters $\kappa_{0}$ and $\beta_{0}$ may assume, we analyze the stability of the solutions in the weak-field limit, similar to that developed in Ref. [33].

As we can see in Eq. (7) the gravitational potential $\phi$ depends on the curvature scalar $R$, such that, any instability in $R$ produces an instability in $\phi$. So, the stability analysis can be performed by perturbing Eq. (5) assuming that

$$
R=R_{0}(r)+\delta R(r, t) .
$$

This perturbation must in principle be induced by a perturbation in $\rho$ such that $\rho \rightarrow \rho_{0}+\delta \rho$. In this case, we have for the perturbed equation

$$
\delta R-\frac{3}{\kappa_{0}}\left[1-\frac{\beta_{0}}{\kappa_{0}}\left(-\partial_{0}^{2}+\nabla^{2}\right)\right]\left(-\partial_{0}^{2}+\nabla^{2}\right) \delta R=8 \pi G \delta \rho .
$$

By taking the Fourier transform of Eq. (23), we have

$$
\delta R_{k}+\frac{3}{\kappa_{0}}\left[1+\frac{\beta_{0}}{\kappa_{0}}\left(\partial_{0}^{2}+k^{2}\right)\right]\left(\partial_{0}^{2}+k^{2}\right) \delta R_{k}=8 \pi G \delta \rho_{k} .
$$

This equation is a nonhomogeneous linear ordinary differential equation, and the stability analysis can be studied by looking only at the homogeneous solution. So ignoring $\delta \rho_{k}$, we have

$$
\frac{3}{\kappa_{0}}\left[1+\frac{\beta_{0}}{\kappa_{0}}\left(\partial_{0}^{2}+k^{2}\right)\right]\left(\partial_{0}^{2}+k^{2}\right) \delta R_{k}+\delta R_{k}=0 .
$$

For $\beta_{0}=0$, we have

$$
\delta \ddot{R}_{k}+\omega_{k}^{2} \delta R_{k}=0
$$

with

$$
\omega_{k}^{2}=k^{2}+\frac{\kappa_{0}}{3}
$$

For $\kappa_{0}>0$, the perturbative solution $\delta R_{k}$ is oscillatory for all the $k$ models and therefore, stable. On the other hand, for $\kappa_{0}<0$ there are unstable $k$ modes that grow exponentially, producing instabilities in the system. 
Let us now look at the case of the complete equation. By manipulating Eq. (25), we can get

$\delta \dddot{R}_{k}+\left(\frac{\kappa_{0}}{\beta_{0}}+2 k^{2}\right) \delta \ddot{R}_{k}+\left(k^{4}+\frac{\kappa_{0}}{\beta_{0}} k^{2}+\frac{\kappa_{0}^{2}}{3 \beta_{0}}\right) \delta R_{k}=0$.

By considering a solution for $\delta R_{k}$ in the form $e^{i \omega t}$, we have

$$
\left[\omega^{4}-\left(\frac{\kappa_{0}}{\beta_{0}}+2 k^{2}\right) \omega^{2}+\left(k^{4}+\frac{\kappa_{0}}{\beta_{0}} k^{2}+\frac{\kappa_{0}^{2}}{3 \beta_{0}}\right)\right] \delta R_{k}=0,
$$

which results in

$$
\sqrt{2} \omega= \pm \sqrt{\left(\frac{\kappa_{0}}{\beta_{0}}+2 k^{2}\right) \pm \sqrt{\left(\frac{\kappa_{0}}{\beta_{0}}+2 k^{2}\right)^{2}-4\left(k^{4}+\frac{\kappa_{0}}{\beta_{0}} k^{2}+\frac{\kappa_{0}^{2}}{3 \beta_{0}}\right)}} .
$$

The stability of the solutions occurs only if the four $\omega$ 's were real for any value of $k$. A necessary, but not sufficient, condition for this to happen is

$$
\frac{\kappa_{0}}{\beta_{0}}+2 k^{2}>0,
$$

which implies that $\kappa_{0}$ and $\beta_{0}$ must have the same sign. If this condition is met, we should still have

$$
0<4\left(k^{4}+\frac{\kappa_{0}}{\beta_{0}} k^{2}+\frac{\kappa_{0}^{2}}{3 \beta_{0}}\right)<\left(\frac{\kappa_{0}}{\beta_{0}}+2 k^{2}\right)^{2} .
$$

For $\beta_{0}$ and $\kappa_{0}$ both negative, the first part of the inequality is not satisfied for all $k$. In fact, with $\beta_{0}<0$, the expression

$$
0<4\left(k^{4}+\frac{\kappa_{0}}{\beta_{0}} k^{2}+\frac{\kappa_{0}^{2}}{3 \beta_{0}}\right),
$$

will be violated with a sufficiently small value of $k$. For $\beta_{0}$ and $\kappa_{0}$ both positive, the first part of the inequality is always satisfied because all the terms of $k^{4}+\left(\kappa_{0} / \beta_{0}\right) k^{2}+\kappa_{0}^{2} / 3 \beta_{0}$ are positive. Furthermore

$$
4\left(k^{4}+\frac{\kappa_{0}}{\beta_{0}} k^{2}+\frac{\kappa_{0}^{2}}{3 \beta_{0}}\right)<\left(\frac{\kappa_{0}}{\beta_{0}}+2 k^{2}\right)^{2} \Rightarrow \beta_{0}<\frac{3}{4} .
$$

Therefore, the stability of the solutions in the weak-field regime only occurs if

$$
\kappa_{0}>0 \text { and } 0 \leq \beta_{0}<\frac{3}{4} .
$$

This analysis excludes oscillatory solutions since it restricts the range of the parameters in $\kappa_{0}>0$ and $0 \leq \beta_{0}<3 / 4$. As seen in the previous section, in this range $l_{ \pm} \in \mathbb{R}$ and the corrections to the gravitational potential are Yukawa-type.

It is interesting to note that this last result can also be obtained by considering a decomposition of the metric in the scalar and massless tensor modes. In order to show that statement, we use some results found in Ref. [19]. In this case, the metric can be decomposed as

$$
h_{\mu \nu}=\tilde{h}_{\mu \nu}-\eta_{\mu \nu} \Phi-\eta_{\mu \nu} \bar{\Phi}
$$

assuming the harmonic gauge

$$
\partial^{\mu} \tilde{\gamma}_{\mu \nu}=0, \text { with } \quad \tilde{\gamma}_{\mu \nu} \equiv \tilde{h}_{\mu \nu}-\frac{1}{2} \eta_{\mu \nu} \tilde{h},
$$

where $\tilde{h}=\eta^{\alpha \beta} \tilde{h}_{\alpha \beta}$. Furthermore, considering the correspondence between the parameters of the theories and the difference of signature of the metric, we obtain the equations

$$
\begin{gathered}
\square \tilde{h}_{\mu \nu}=0, \\
\left(\square-m_{+}^{2}\right) \Phi=0, \\
\left(\square-m_{-}^{2}\right) \bar{\Phi}=-m_{+}^{2} \Phi,
\end{gathered}
$$

where for the scalar equations

$$
m_{ \pm}^{2}=\frac{\kappa_{0}}{2 \beta_{0}}\left(1 \pm \sqrt{1-\frac{4 \beta_{0}}{3}}\right) .
$$

For we have $m_{ \pm}^{2}>0$, we conclude that $\kappa_{0}$ and $\beta_{0}$ must be restricted in the intervals expressed in relation (33). Finally, note that the conditions that lead to $m_{ \pm}^{2}>0$, i.e., the absence of tachyons are the same that guarantee the stability of the weak-field regime solutions.

\section{BLACK HOLE SOLUTIONS}

In recent years, there has been growing interest in investigating black hole solutions in higher-order theories of gravity [27-32, 34-41]. Such analyzes allow us to better understand, in addition to the structure and nature of the solutions, these theories in strong gravitational field regimes. We know that the spherically symmetric and static solution in a vacuum of GR is the Schwarzschild one. Furthermore, Birkhoof's theorem tells us that it is the only one. In this section, we investigate the existence of exterior non-Schwarzschild black hole solutions, and we discuss under what conditions they may be physically achievable.

By performing this analysis, we use an approach called the Lichnerovicz method in the literature, similar to what is done in Refs. [27-29]. In a nutshell, this methodology informs us about the possibility of the exterior Schwarzschild solution to be unique - which is interesting in our case, since $R_{\mu \nu}=$ 0 , that is, the Schwarzschild solution, is a solution of field equations (2) in a vacuum.

We start this investigation, considering the line element due to a spherically symmetric and static object, which is written as

$$
d s^{2}=g_{00} d t^{2}+g_{11} d r^{2}+r^{2}\left(d \theta^{2}+\sin ^{2} \theta d \phi^{2}\right),
$$

where $g_{00}$ and $g_{11}$ are functions only of the radial coordinate. Then let us take the trace of the field equations (2) in a vacuum, multiply it by the curvature scalar $R$ and use the Leibniz rule a few times to obtain total derivative terms. That gives us 


$$
\begin{gathered}
R^{2}+\frac{3}{\kappa_{0}} \nabla_{\mu} R \nabla^{\mu} R-\frac{3 \beta_{0}}{2 \kappa_{0}^{2}} R \nabla_{\mu} R \nabla^{\mu} R+\frac{3 \beta_{0}}{\kappa_{0}^{2}} \square R \square R-\frac{\alpha_{0}}{3 \kappa_{0}^{2}} R^{4}+\frac{3 \alpha_{0}}{\kappa_{0}^{2}} \nabla_{\mu} R \nabla^{\mu} R^{2}+ \\
-\frac{3}{\kappa_{0}} \nabla_{\mu}\left(R \nabla^{\mu} R\right)+\frac{3 \beta_{0}}{\kappa_{0}^{2}} \nabla_{\mu}\left(R \nabla^{\mu} \square R\right)-\frac{3 \beta_{0}}{\kappa_{0}^{2}} \nabla_{\mu}\left(\nabla^{\mu} R \square R\right)+\frac{\beta_{0}}{\kappa_{0}^{2}} \nabla_{\mu}\left(R^{2} \nabla^{\mu} R\right)-\frac{3 \alpha_{0}}{\kappa_{0}^{2}} \nabla_{\mu}\left(R \nabla^{\mu} R^{2}\right)=0 .
\end{gathered}
$$

Now, integrating Eq. (41) into the outside region of the

horizon $r_{h}$, where $g_{00}<0$ and $g_{11}>0$, we obtain

$$
\begin{gathered}
\int d^{4} x \sqrt{-g}\left[R^{2}+\frac{3 g^{11}}{\kappa_{0}}\left(1-\frac{\beta_{0}}{2 \kappa_{0}} R+\frac{2 \alpha_{0}}{\kappa_{0}} R\right)\left(\partial_{1} R\right)^{2}+\frac{3 \beta_{0}}{\kappa_{0}^{2}}(\square R)^{2}-\frac{\alpha_{0}}{3 \kappa_{0}^{2}} R^{4}\right]+ \\
-\frac{3}{\kappa_{0}} K\left(\int_{r_{h}}+\int_{\infty}\right) d S_{1}\left(r^{2} \sqrt{-g_{00} g^{11}} R \partial_{1} R\right)+\frac{3 \beta_{0}}{\kappa_{0}^{2}} K\left(\int_{r_{h}}+\int_{\infty}\right) d S_{1}\left[r^{2} \sqrt{-g_{00} g^{11}} R \partial_{1}(\square R)\right]+ \\
-\frac{3 \beta_{0}}{\kappa_{0}^{2}} K\left(\int_{r_{h}}+\int_{\infty}\right) d S_{1}\left(r^{2} \sqrt{-g_{00} g^{11}} \partial_{1} R \square R\right)+ \\
+\frac{\beta_{0}}{\kappa_{0}^{2}} K\left(\int_{r_{h}}+\int_{\infty}\right) d S_{1}\left(r^{2} \sqrt{-g_{00} g^{11}} R^{2} \partial_{1} R\right)-\frac{3 \alpha_{0}}{\kappa_{0}^{2}} K\left(\int_{r_{h}}+\int_{\infty}\right) d S_{1}\left(r^{2} \sqrt{-g_{00} g^{11}} g^{11} R \partial_{1} R^{2}\right)=0,
\end{gathered}
$$

where the chosen notation in the second, third and fourth lines means that the integration is evaluated at the spherically symmetric hypersurfaces $r=r_{h}$ and $r \rightarrow \infty$. Also, $K$ is a constant term involving angular and temporal coordinates and

$$
\square R=\frac{1}{r^{2} \sqrt{-g_{00} g_{11}}} \partial_{1}\left(r^{2} \sqrt{-g_{00} g_{11}} g^{11} \partial_{1} R\right) .
$$

If we consider that the curvature scalar and its derivatives up to the third-order are well-behaved quantities at $r=r_{h}$, which is reasonable since the event horizon $r_{h}$ is an apparent singularity, we have that the associated surface terms are null. This occurs because, by definition, an event horizon is a null hypersurface in which $g^{11}\left(r_{h}\right)=g_{00}\left(r_{h}\right)=0$ [42]. In turn, the surface terms evaluated at infinity also vanish, since in this region far from the origin, the expressions for the weak-field regime are valid, so that the stable Yukawa-type solutions decay faster than any powers in $r$. Thus, we get

$$
\int d^{4} x \sqrt{-g}\left[R^{2}+\frac{3 g^{11}}{\kappa_{0}}\left(1-\frac{\beta_{0}}{2 \kappa_{0}} R+\frac{2 \alpha_{0}}{\kappa_{0}} R\right)\left(\partial_{1} R\right)^{2}+\frac{3 \beta_{0}}{\kappa_{0}^{2}}(\square R)^{2}-\frac{\alpha_{0}}{3 \kappa_{0}^{2}} R^{4}\right]=0 .
$$

First, note that by making $\alpha_{0}=\beta_{0}=0$, which represents the gravity with quadratic correction in the curvature scalar, we have

$$
\int d^{4} x \sqrt{-g}\left[R^{2}+\frac{3 g^{11}}{\kappa_{0}}\left(\partial_{1} R\right)^{2}\right]=0
$$

Since $\kappa_{0}$, as well as $g^{11}$, is a positive quantity and $R^{2}$ and $\left(\partial_{1} R\right)^{2}$ are quadratic terms, we note that to satisfy the relation (45), both terms must vanish independently, in such a way that it gives $R=0$. By substituting this result in Eq. (2) free of source (with $\alpha_{0}=\beta_{0}=0$ ), we find that $R_{\mu \nu}=0$. Thus, the Schwarzschild outer solution is the unique spherically symmetric and static solution of the gravity with quadratic correction in the curvature scalar.
Similarly, by following the same approach for the full relation (44), we observe initially that the first and third terms are non-negative, since we saw in the last section that $\beta_{0}>0$. So, for the only spherically symmetric solution of the field equations (2) in vacuum to be the Schwarzschild solution, it is necessary that $\alpha_{0} \leq 0$ and that the curvature scalar outside the horizon $R_{\text {out }}$ satisfies

$$
R_{\text {out }} \leq \frac{2 \kappa_{0}}{4\left|\alpha_{0}\right|+\beta_{0}}
$$

If these two conditions are fulfilled, all terms in Eq. (44) will be non-negative, and to satisfy it, each term must vanish independently. Then, we conclude that $R_{\text {out }}=0$. Based on (46), we can state that if $R_{\text {out }}$ is less than or equal to such a quantity, then it will necessarily be zero in that region. Unfortunately, 
since we cannot guarantee that $R_{\text {out }}=0$ unrestrictedly, the Lichnerovicz method is quite inconclusive in discriminating if the Schwarzschild solution is the only one.

On the other hand, we can also state that if $R_{\text {out }}$ exceeds this amount, non-Schwarzschild black hole solutions might exist. Then let us estimate the order of magnitude of $R_{\text {out }}$ for this to occur. The first consideration to be made is that $\left(4\left|\alpha_{0}\right|+\beta_{0}\right) \sim \beta_{0}$ in (46). This is justified since $R^{3}$ and $R \square R$ are second-order correction terms, and therefore, $\alpha_{0}$ and $\beta_{0}$ are expected to have similar magnitudes. Besides that, the stability analysis of the weak-field limit solutions shows us that there is an upper limit for the parameter $\beta_{0}$, namely the value of $3 / 4$, which leads to a stable gravitational potential. At the limit where $\beta_{0}$ assumes such a value, it follows that

$$
R_{\text {out }} \gtrsim \frac{8 \kappa_{0}}{3} .
$$

If we assume that the additional terms in besides to the Einstein-Hilbert in the action (1) are classical corrections from a quantum theory of gravity, it is reasonable to expect that $\kappa_{0} \sim M_{P}^{2}$. Thus,

$$
R_{\text {out }} \gtrsim \frac{8 \kappa_{0}}{3} \sim 10^{36} \mathrm{GeV}^{2} .
$$

Another possibility is suppose that cosmic inflation is generated by the modified gravity (1). In this case, we can establish the order of magnitude of $R_{\text {out }}$ based on the energy scale of inflation. By using the results presented in Ref. [24], namely,

$$
\kappa_{0} \approx 6 \pi^{2} A_{s} r M_{P}^{2} \sim 10^{-9} M_{P}^{2} \text { and } \beta_{0}^{\max } \sim 10^{-2},
$$

where the scalar amplitude $A_{s} \approx 1.96 \times 10^{-9}$ [43] and the tensor-to-scalar ratio $r \sim 10^{-2}$ [24], we were able to estimate that

$$
R_{\text {out }} \gtrsim \frac{2 \kappa_{0}}{\beta_{0}^{\max }} \sim \frac{10^{27}}{10^{-2}}=10^{29} \mathrm{GeV}^{2} .
$$

Thus, non-Schwarzschild black hole solutions may exist only for values of $R_{\text {out }}$ which exceed $10^{29} \mathrm{GeV}^{2}$. Very roughly, we can estimate the order of magnitude of the horizon extrapolating the validity of the weak-field results. Using Eqs. (8), (16), (49) and imposing the relation (50), we obtain

$$
\frac{G M}{r}\left(e^{-\frac{r}{l_{+}}}-e^{-\frac{r}{l_{-}}}\right) \gtrsim 10^{2},
$$

where $l_{ \pm}$is given by Eq. (15). So, due to the exponential decay, the necessary but not sufficient condition for $R_{\text {out }} \gtrsim$ $10^{29} \mathrm{GeV}^{2}$ is

$$
r \lesssim l_{ \pm} \sim \sqrt{\frac{3}{2 \kappa_{0}}} \Rightarrow r \lesssim 10^{4} M_{P}^{-1} \sim 10^{-31} \mathrm{~m} .
$$

As it is expected that $R_{\text {out }}$ reaches its maximum value near to the horizon, we get

$$
R_{\text {out }} \gtrsim 10^{29} \mathrm{GeV}^{2} \Rightarrow r_{h} \lesssim 10^{-31} \mathrm{~m} .
$$

The previous analysis is quite rough and the estimate of $r_{h}$ can vary a few orders of magnitude. Nevertheless, even considering this variation, it is clear that the existence of nonSchwarzschild solutions will not occur in the usual astrophysical context. At least for the values of $\kappa_{0}$ considered.

\section{A. Deviations from the Schwarzschild solution}

We saw in the previous section that the use of the Lichnerovicz method proved inconclusive in giving us an answer to the question of whether or not there are exterior nonSchwarzschild solutions. We have also seen, by making use of some estimations and extrapolations, that such solutions may be manifested only in black holes whose horizons are extremely small. We now want to investigate whether there are spherically symmetric and static solutions in the vacuum which can be obtained from a continuous deformation of the Schwarzschild solution, similar to that developed in Ref. [27].

First of all, let's see how the trace of the field equations (2) in the vacuum behaves for the case where the curvature scalar $R$ deviates infinitesimally from the Schwarzschild solution i.e.,

$$
R(r)=\varepsilon f(r),
$$

with $\varepsilon \ll 1$. Discarding terms beyond the first-order in $\varepsilon$, we have

$$
-f+\frac{3}{\kappa_{0}} \square_{S c h} f-3 \frac{\beta_{0}}{\kappa_{0}^{2}} \square_{S c h}^{2} f=0,
$$

where $\square_{S c h}$ is constructed with the Schwarzschild metric. Writing explicitly the last two terms in Eq. (54), we obtain

$$
\begin{gathered}
-f+\left\{\frac{3}{\kappa_{0}} \frac{1}{r^{2}}\left[2 r\left(1-\frac{r_{h}}{r}\right)+r_{h}\right]+\frac{12 \beta_{0}}{\kappa_{0}^{2}} \frac{r_{h}}{r^{4}}\left(1-\frac{r_{h}}{r}\right)\right\} \frac{d f}{d r}+ \\
+\left\{\frac{3}{\kappa_{0}}\left(1-\frac{r_{h}}{r}\right)-\frac{6 \beta_{0}}{\kappa_{0}^{2}} \frac{1}{r^{2}}\left[1-\left(1-\frac{r_{h}}{r}\right)^{2}\right]\right\} \frac{d^{2} f}{d r^{2}}+ \\
-\frac{6 \beta_{0}}{\kappa_{0}^{2}}\left[\frac{1}{r}\left(1-\frac{r_{h}}{r}\right)^{2}+\frac{1}{r}\left(1-\frac{r_{h}}{r}\right)+\frac{r_{h}}{r^{2}}\left(1-\frac{r_{h}}{r}\right)\right] \frac{d^{3} f}{d r^{3}}+ \\
-\frac{3 \beta_{0}}{\kappa_{0}^{2}}\left(1-\frac{r_{h}}{r}\right)^{2} \frac{d^{4} f}{d r^{4}}=0 .
\end{gathered}
$$

From the Eq. (55), we make a construction similar to that one developed in the previous section based on the Lichnerovicz method. The idea is to set up a quadratic relation for $f$, integrate it outside the horizon $r_{h}$, and check if we can conclude, through the independently vanishing of each of the terms, that $f$ is null in that region. In this sense, by multiplying Eq. (55) by $r^{5}$, we can express it in the form

$$
h_{0} f+h_{1} f^{\prime}+h_{2} f^{\prime \prime}+h_{3} f^{(3)}+h_{4} f^{(4)}=0,
$$


where

$$
\begin{gathered}
h_{0}=-r^{5}, \\
h_{1}=\frac{3}{\kappa_{0}} r^{3}\left(2 r-r_{h}\right)+\frac{12 \beta_{0}}{\kappa_{0}^{2}} r_{h}\left(r-r_{h}\right), \\
h_{2}=\frac{3}{\kappa_{0}} r^{4}\left(r-r_{h}\right)-\frac{6 \beta_{0}}{\kappa_{0}^{2}} r\left[r^{2}-\left(r-r_{h}\right)^{2}\right], \\
h_{3}=-\frac{12 \beta_{0}}{\kappa_{0}^{2}} r^{3}\left(r-r_{h}\right), \\
h_{4}=-\frac{3 \beta_{0}}{\kappa_{0}^{2}} r^{3}\left(r-r_{h}\right)^{2} .
\end{gathered}
$$

By multiplying Eq. (56) by $u(r) f$, where $u$ is a function whose form will be obtained later, we have

$$
h_{0} u f^{2}+h_{1} u f f^{\prime}+h_{2} u f f^{\prime \prime}+h_{3} u f f^{(3)}+h_{4} u f f^{(4)}=0 .
$$

Now, performing a series of derivative manipulations in Eq. (57), it is possible to rewrite it in terms of the square of the functions $f, f^{\prime}, f^{\prime \prime}$, and total derivative terms, as follows

$$
\begin{gathered}
h_{0} u f^{2}+\left[-h_{2} u+\frac{3}{2}\left(h_{3} u\right)^{\prime}-2\left(h_{4} u\right)^{\prime \prime}\right] f^{\prime 2}+h_{4} u f^{\prime \prime 2}+ \\
+\left[h_{1} u-\left(h_{2} u\right)^{\prime}+\left(h_{3} u\right)^{\prime \prime}-\left(h_{4} u\right)^{(3)}\right] f f^{\prime}+ \\
+\left(h_{2} u f f^{\prime}\right)^{\prime}+\left(h_{3} u f f^{\prime \prime}\right)^{\prime}-\frac{1}{2}\left(h_{3} u f^{\prime 2}\right)^{\prime}+ \\
-\left(\left(h_{3} u\right)^{\prime} f f^{\prime}\right)^{\prime}-\left(h_{4} u f^{\prime} f^{\prime \prime}\right)^{\prime}+\left(h_{4} u f f^{(3)}\right)^{\prime}+ \\
-\left(\left(h_{4} u\right)^{\prime} f f^{\prime \prime}\right)^{\prime}+\left(\left(h_{4} u\right)^{\prime} f^{\prime 2}\right)^{\prime}+\left(\left(h_{4} u\right)^{\prime \prime} f f^{\prime}\right)^{\prime}=0 .
\end{gathered}
$$

Then, we choose the form of $u(r)$ so that the quantity in brackets accompanying the cross term $f f^{\prime}$ is canceled, which is,

$$
h_{1} u-\left(h_{2} u\right)^{\prime}+\left(h_{3} u\right)^{\prime \prime}-\left(h_{4} u\right)^{(3)}=0 \Rightarrow u \propto 1 / r^{3} .
$$

By integrating Eq. (58) in the region outside the horizon, we obtain that

$$
\begin{aligned}
\int\left\{r^{2} f^{2}\right. & \left.+\left[\frac{3}{\kappa_{0}} r\left(r-r_{h}\right)+\frac{6 \beta_{0}}{\kappa_{0}^{2}} \frac{\left(r-r_{h}\right)^{2}}{r^{2}}\right] f^{\prime 2}\right\} d r+ \\
+ & \frac{3 \beta_{0}}{\kappa_{0}^{2}} \int\left(r-r_{h}\right)^{2} f^{\prime \prime 2} d r+I_{S}=0
\end{aligned}
$$

where $I_{S}$ represents the integral of all surface terms

$$
\begin{aligned}
I_{S}= & \int_{r_{h}}^{\infty}\left[\left(\frac{h_{2}}{r^{3}} f f^{\prime}\right)^{\prime}+\left(\frac{h_{3}}{r^{3}} f f^{\prime \prime}\right)^{\prime}-\frac{1}{2}\left(\frac{h_{3}}{r^{3}} f^{\prime 2}\right)^{\prime}\right] d r+ \\
& -\int_{r_{h}}^{\infty}\left[\left(\left(\frac{h_{3}}{r^{3}}\right)^{\prime} f f^{\prime}\right)^{\prime}+\left(\frac{h_{4}}{r^{3}} f^{\prime} f^{\prime \prime}\right)^{\prime}\right] d r+ \\
& +\int_{r_{h}}^{\infty}\left[\left(\frac{h_{4}}{r^{3}} f f^{(3)}\right)^{\prime}-\left(\left(\frac{h_{4}}{r^{3}}\right)^{\prime} f f^{\prime \prime}\right)^{\prime}\right] d r+ \\
& +\int_{r_{h}}^{\infty}\left[\left(\left(\frac{h_{4}}{r^{3}}\right)^{\prime} f^{\prime 2}\right)^{\prime}+\left(\left(\frac{h_{4}}{r^{3}}\right)^{\prime \prime} f f^{\prime}\right)^{\prime}\right] d r
\end{aligned}
$$

The integration of the various surface terms evaluated at infinity vanishes out, since in that region $f$ and its first derivatives have a Yukawa-like behavior, decaying faster than any polynomial term. In turn, in order to show that the surface terms cancel each other on the horizon, we only need to consider that the function $f$ and its first derivatives are well behaved at $r=r_{h}$. With that in mind, it is easy to show that at the horizon,

$$
\begin{aligned}
I= & -\left(-\frac{6 \beta_{0}}{\kappa_{0}^{2}} f f^{\prime}\right)-\left((0) f f^{\prime \prime}\right)+\frac{1}{2}\left((0) f^{\prime 2}\right)+ \\
+ & \left(-\frac{12 \beta_{0}}{\kappa_{0}^{2}} f f^{\prime}\right)+\left((0) f^{\prime} f^{\prime \prime}\right)-\left((0) f f^{(3)}\right)+ \\
& +\left((0) f f^{\prime \prime}\right)-\left((0) f^{\prime 2}\right)-\left(-\frac{6 \beta_{0}}{\kappa_{0}^{2}} f f^{\prime}\right),
\end{aligned}
$$

and therefore

$$
I=0 .
$$

Let us turn our attention back to Eq. (60). We observe that outside the horizon $\left(r>r_{h}\right)$ each of the coefficients that accompany the quadratic terms are non-negative. We see then that the only way for Eq. (60) to be satisfied is if each of the terms is null independently, which is, if $f=f_{\text {out }}=0$. This is an interesting result because it means that an infinitesimal perturbation of the Schwarzschild exterior solution does not exist, at least not in the first-order.

The next step is to analyse higher-order perturbations of the Schwarzschild solution. For this purpose, we can represent a perturbation from the Schwarzschild solution by

$$
g_{\mu \nu}=g_{\mu \nu}^{(0)}+\varepsilon g_{\mu \nu}^{(1)}+\varepsilon^{2} g_{\mu \nu}^{(2)}+\cdots,
$$

where $\varepsilon$ is the parameter of the perturbation and $g_{\mu \nu}^{(0)}$ is the Schwarzschild metric. Such perturbation represented in Eq. (63) induces a perturbation in the curvature scalar $R$ given by

$$
R=R^{(0)}+\varepsilon R^{(1)}+\varepsilon^{2} R^{(2)}+\cdots,
$$

in which $R^{(0)}=R_{S c h}=0$, and from the previous analysis, $R_{\text {out }}^{(1)} \equiv f_{\text {out }}=0$. Taking the trace of the field equation (2) in the vacuum and considering terms up to the second-order of perturbation, we obtain

$$
-R_{\text {out }}^{(2)}+\frac{3}{\kappa_{0}} \square_{S c h} R_{\text {out }}^{(2)}-3 \frac{\beta_{0}}{\kappa_{0}^{2}} \square_{S c h}^{2} R_{\text {out }}^{(2)}=0 .
$$

The relation (65) is the same for the first-order perturbation $f$, namely (54), whose result we already know: it vanishes outside the horizon. Then we can conclude that there is no $R_{\text {out }}^{(2)}$ perturbation. Extrapolating this result it is possible to show that higher-order perturbations $R_{\text {out }}^{(n)}$ are all null.

Based on the previous analysis, we could think that the result $R_{\text {out }}^{(n)}=0$ is valid for any $r_{h}$, but this is not true. The main point is that the perturbative approach developed here configure a regular perturbation analysis, and formally, this kind of analysis cannot be employed in our case because the perturbative terms are differential high-order terms. In this situation, 
the correct approach is to use singular perturbation analysis (see Refs. [44, 45] for an introduction). However, it is safe to employ usual regular perturbation if the region of interest is far from the boundary layer region. For our model, the boundary layer is located near to the origin and its thickness can be estimated as $\kappa_{0}^{-1 / 2}$. As we are interested in outside horizon solutions, our result, based on regular perturbative method, remain valid for $r_{h} \gg \kappa_{0}^{-1 / 2}$. Therefore, we conclude that there are no deviations from the Schwarzschild solution outside the horizon, since $r_{h} \gg \kappa_{0}^{-1 / 2}$. This result is in agreement to the conclusion presented in the previous section.

\section{FINAL COMMENTS}

In this paper we have studied spherically symmetric and static solutions in the weak-field regime and black hole context of a gravity theory which includes all the curvature scalar terms up to the second-order corrections.

In the weak-field regime, we developed a complete study for the proposed gravity, in which we obtained all possible solutions due to a point mass. In addition, we verified that among all found solutions only those which present a Yukawatype behaviour $\left(\kappa_{0}>0\right.$ and $\left.0 \leq \beta_{0}<3 / 4\right)$ are stable when a temporal perturbation is performed. It is worth mentioning, that the same restriction for $\kappa_{0}$ and $\beta_{0}$ emerges if we consider that the inflationary regime is generated by the action (1) with $\alpha_{0}=0$ [24]. In fact, the constraints $\kappa_{0}>0$ and $0 \leq \beta_{0}<3 / 4$ are necessary conditions to ensure that the inflation properly end in a graceful exit. The consistency between these two results is not completely unexpected as both address stability issues. Nevertheless, their coherence provides an important clue in constraining effective theories of quantum gravity.

Motivated by the growing interest in black hole solutions in higher-order gravities, see Refs. [27-32, 34-41], we also developed an analysis of spherically symmetric and static black holes solutions. In this investigation, we used the Lichnerovicz method to investigate the possibility of existence of exterior non-Schwarzschild solutions. In both approaches used, namely, 1) considering the trace equation of the full theory and 2) assuming a continuous deformation from the exterior Schwarzschild solution, our results suggest the absence of non-Schwarzschild macroscopical black holes. This is due to the fact that the curvature scalar $R_{\text {out }}$ must exceed, in the most conservative estimate, $10^{29} \mathrm{GeV}^{2}$ for the existence of non-Schwarzschild solutions, and this implies in black holes whose horizons are smaller or of the order of $10^{-31} \mathrm{~m}$. In this context, there are two main questions which should be addressed. First, do these mini black holes actually exist, since $R_{\text {out }}>10^{29} \mathrm{GeV}^{2}$ is a necessary but not sufficient condition for its existence? Second, how do they behave under Hawking radiation (see Ref. [46] and references therein), and consequently, what would be the lifetime of these objects? These two issues will be addressed by the authors in a future work.

\section{ACKNOWLEDGMENTS}

G. Rodrigues-da-Silva thanks CAPES/UFRN-RN (Brazil) for financial support and L. G. Medeiros acknowledge CNPq (Brazil) for partial financial support.
[1] B. P. Abbott et al. (LIGO Scientific, Virgo), Phys. Rev. Lett. 116, 061102 (2016), arXiv:1602.03837 [gr-qc].

[2] B. P. Abbott et al. (LIGO Scientific, Virgo), Phys. Rev. Lett. 116, 221101 (2016), [Erratum: Phys. Rev. Lett.121,no.12,129902(2018)], arXiv:1602.03841 [gr-qc].

[3] B. P. Abbott et al. (LIGO Scientific Collaboration and Virgo Collaboration), Phys. Rev. Lett. 119, 161101 (2017).

[4] G. 't Hooft and M. Veltman, Annales de l'I.H.P. Physique théorique 20, 69 (1974).

[5] R. Utiyama and B. S. DeWitt, Journal of Mathematical Physics 3, 608 (1962), https://doi.org/10.1063/1.1724264.

[6] K. S. Stelle, Phys. Rev. D 16, 953 (1977).

[7] R. P. Woodard, ArXiv e-prints arXiv: 1506.02210 [hep-th].

[8] M. Asorey, J. L. Lopez, and I. L. Shapiro, Int. J. Mod. Phys. A12, 5711 (1997), arXiv:hep-th/9610006 [hep-th].

[9] I. L. Shapiro, Class. Quant. Grav. 25, 103001 (2008), arXiv:0801.0216 [gr-qc].

[10] A. Accioly, J. Helayël-Neto, E. Scatena, and R. Turcati, International Journal of Modern Physics D 22, 1342015 (2013).

[11] L. Modesto, Nucl. Phys. B909, 584 (2016), arXiv:1602.02421 [hep-th].
[12] T. D. Lee and G. C. Wick, Phys. Rev. D 2, 1033 (1970).

[13] J. F. Donoghue and G. Menezes, Phys. Rev. D100, 105006 (2019), arXiv:1908.02416 [hep-th].

[14] E. T. Tomboulis, (1997), arXiv:hep-th/9702146 [hep-th].

[15] L. Modesto, Phys. Rev. D86, 044005 (2012), arXiv:1107.2403 [hep-th].

[16] T. Biswas, E. Gerwick, T. Koivisto, and A. Mazumdar, Phys. Rev. Lett. 108, 031101 (2012), arXiv:1110.5249 [gr-qc].

[17] I. L. Shapiro, Phys. Lett. B744, 67 (2015), arXiv:1502.00106 [hep-th].

[18] I. Quandt and H.-J. Schmidt, Astron. Nachr. 312, 97 (1991), arXiv:gr-qc/0109005 [gr-qc].

[19] A. Accioly, B. L. Giacchini, and I. L. Shapiro, Phys. Rev. D96, 104004 (2017), arXiv:1610.05260 [gr-qc].

[20] B. L. Giacchini and T. de Paula Netto, Eur. Phys. J. C79, 217 (2019), arXiv:1806.05664 [gr-qc].

[21] R. R. Cuzinatto, C. A. M. de Melo, L. G. Medeiros, and P. J. Pompeia, Eur. Phys. J. C53, 99 (2008), arXiv:gr-qc/0611116 [gr-qc].

[22] R. R. Cuzinatto, C. A. M. de Melo, L. G. Medeiros, and P. J. Pompeia, Phys. Rev. D93, 124034 (2016), [Erratum: Phys. Rev.D98,no.2,029901(2018)], arXiv:1603.01563 [gr-qc].

[23] A. R. R. Castellanos, F. Sobreira, I. L. Shapiro, and A. A. Starobinsky, JCAP 1812, 007 (2018), arXiv:1810.07787 [gr-qc]. 
[24] R. R. Cuzinatto, L. G. Medeiros, and P. J. Pompeia, JCAP 1902, 055 (2019), arXiv:1810.08911 [gr-qc].

[25] R. R. Cuzinatto, C. A. M. de Melo, L. G. Medeiros, and P. J. Pompeia, Phys. Rev. D99, 084053 (2019), arXiv:1806.08850 [gr-qc].

[26] K. S. Stelle, Gen. Rel. Grav. 9, 353 (1978).

[27] H. Lü, A. Perkins, C. N. Pope, and K. S. Stelle, Phys. Rev. D 92, 124019 (2015).

[28] W. Nelson, arXiv:1010.3986 [gr-qc].

[29] H. Lu, A. Perkins, C. N. Pope, and K. S. Stelle, Phys. Rev. Lett. 114, 171601 (2015), arXiv:1502.01028 [hep-th].

[30] J. Podolsky, R. Svarc, V. Pravda, and A. Pravdova, Phys. Rev. D98, 021502 (2018), arXiv:1806.08209 [gr-qc].

[31] J. Podolský, R. Švarc, V. Pravda, and A. Pravdova, Phys. Rev. D101, 024027 (2020), arXiv:1907.00046 [gr-qc].

[32] K. Kokkotas, R. A. Konoplya, and A. Zhidenko, Phys. Rev. D96, 064007 (2017), arXiv:1705.09875 [gr-qc].

[33] L. Perivolaropoulos and L. Kazantzidis, Int. J. Mod. Phys. D28, 1942001 (2019), arXiv:1904.09462 [gr-qc].

[34] R. A. Hennigar and R. B. Mann, Phys. Rev. D 95, 064055 (2017), arXiv:1610.06675 [hep-th].

[35] P. Bueno and P. A. Cano, Phys. Rev. D 94, 124051 (2016), arXiv:1610.08019 [hep-th].
[36] K. Goldstein and J. J. Mashiyane, Phys. Rev. D97, 024015 (2018), arXiv:1703.02803 [hep-th].

[37] P. Bueno and P. A. Cano, Class. Quant. Grav. 34, 175008 (2017), arXiv:1703.04625 [hep-th].

[38] R. A. Hennigar, D. Kubiz $ª ́ k$ and R. B. Mann, Phys. Rev. D 95, 104042 (2017), arXiv:1703.01631 [hep-th].

[39] J. Ahmed, R. A. Hennigar, R. B. Mann, and M. Mir, JHEP 05, 134 (2017), arXiv:1703.11007 [hep-th].

[40] P. Bueno and P. A. Cano, Phys. Rev. D 96, 024034 (2017), arXiv: 1704.02967 [hep-th].

[41] E. Hernandéz-Lorenzo and C. F. Steinwachs, (2020), arXiv:2003.12109 [gr-qc].

[42] J. D. Bekenstein, Phys. Rev. D5, 1239 (1972).

[43] Y. Akrami et al. (Planck), arXiv:1807.06211 [astro-ph.CO].

[44] R. Johnson, Singular perturbation theory: techniques with applications to engineering, 1st ed., Mathematical and Analytical Techniques with Applications to Engineering (Springer, 2004).

[45] M. H. Holmes, Introduction to Perturbation Methods, 2nd ed., Texts in Applied Mathematics (Springer, 2012).

[46] R. A. Konoplya and A. F. Zinhailo, Phys. Rev. D99, 104060 (2019), arXiv:1904.05341 [gr-qc]. 